RESEARCH ARTICLE

\title{
Prevalence of Staphylococci in Commercially Processed Food Products in Karachi-Pakistan
}

\author{
Zulfiqar Ali Mirani, Afsheen Aqeel, Shagufta Naz, Anila Siddiqi, Mohammad Naseem Khan, Seema Ismat \\ Khan
}

Microbiology Analytical Centre, PCSIR Laboratories Complex, Karachi-Pakistan

\begin{abstract}
Objectives: This study was designed to determine the prevalence of Staphylococci, Staphylococcus aureus ( $S$. aureus), and Methicillin Resistant $S$. aureus (MRSA) in commercially prepared food items that involve human handling, in Karachi-Pakistan.
\end{abstract}

Methods: In a cross-sectional survey approach, in total 1012 food samples were analyzed. These samples were provided by different food processing industries during 2013 to 2016. Barid-Parker agar plates with egg yolk tellurite (Oxoid) along with Mannitol Salt Agar (BioM), Staph-chromo agar (Merck), Staphylococcus 110 Agar (BioM), and Blood Agar (Oxoid) were used for isolation and identification of Staphylococci. Polymerase chain reaction (PCR) was used for amplification of $m e c A$ gene and specie identification via 16 s RNA.

Results: Total 723 samples $(71.4 \%)$ showed the presence of Staphylococci. Out of 723 staphylococcal isolates, 367 $(36.2 \%)$ were $S$. aureus and $85(8.3 \%)$ isolates were confirmed as MRSA. Molecular studies for MRSA typing showed that most of the isolates (74.1\%) belong to SCCmecA IV and 20\% MRSA isolates were SCCmecA type II and $5.8 \%$ isolates carried SCCmecA type III. The MRSA isolates of SCCmecA type II belong to agr type I, while majority (67\%) of the isolates carry agr type II.

Conclusion: This study suggested that majority of MRSA isolates recovered from commercial food items belongs to SCCmecA IV and human handling is a major factor for introduction of staphylococci in processed food items. $J$ Microbiol Infect Dis 2017; 7(2): 83-87

Keywords: Staphylococci, S. aureus, Methicillin Resistant S. aureus

\section{INTRODUCTION}

Staphylococci are gram positive bacteria, known to survive under a wide range of environments e.g. on dry surfaces, high salt concentration and hospital set-ups $[1,2]$. Staphylococci exist in air, dust, sewage, water, milk, and food or on food equipment, environmental surfaces, humans, and animals. Humans and animals are the primary reservoirs [1]. Mostly, it colonizes persons in hospital as well as community setups and is transmitted via person to person contact as well as through inanimate objects [2]. The food borne cases of Staphylococci have been reported from all over the world [2]. Presence of pathogens in food products imposes potential hazard for consumers and causes grave economic loss via food-borne disease [3]. Staphylococci enter in a food set-up and contaminate the food products via human handlers or healthy nasal carriers. After entry, it multiplies in food and produce toxins that can cause food poisoning, a gastrointestinal illness $[2,3]$. Food items made by hands without cooking are at high risk to carry Staphylococcal toxins. However air, dust, and food contact surfaces may also serve as vehicles in the transfer of Staphylococci in foods. Staphylococcus aureus is one of the well-known pathogen of Staphylococci family [1-3]. It tolerates high salt concentration; desiccation and can grow in a wide range of temperatures ( 7 ${ }^{\circ} \mathrm{C}$ to $48.5^{\circ} \mathrm{C}$; optimum 30 to $37^{\circ} \mathrm{C}$ ), $\mathrm{pH}(4.2$ to 9.3; optimum 7 to 7.5). These characteristics favor growth of the organism in many food products [3]. The highly resistant and pathogenic

\footnotetext{
Correspondence: Dr. Zulfiqar Ali Mirani, Senior Scientific Officer, PCSIR Laboratories Complex Karachi-Pakistan E-mail:mirani_mrsa@yahoo.com

Received: 28 June 2016, Accepted: 09 February 2017

Copyright (C JMID / Journal of Microbiology and Infectious Diseases 2017, All rights reserved
} 
strain among Staphylococci is Methicillin Resistant S. aureus (MRSA). Originally, MRSA was found in hospital set-ups; in recent times it is frequently reported in the community as well, and considered as a source of food borne illness [4]. Food borne MRSA isolates are resistant to penicillin and oxacillin, only, whereas hospital isolates of MRSA are multi-drug resistant hence are difficult to control [4]. In S. aureus Methicillin resistance is mediated by $m e c A$ gene, which is located on mobile genetic element known as staphylococcal cassette chromosome mec (SCCmec). There are five major types of SCCmec elements (I-V). The majority of hospital-acquired MRSA strains carry SCCmec types I, II, or III, whereas community-acquired MRSA strains carry SCCmec types IV or V $[8,9]$. World Health Organization (WHO) defines foodborne disease as "disease of infectious or toxic nature caused by the consumption of food or water" [3]. S. aureus is one of the major pathogens responsible for food borne infections, world-wide. Present study describes the prevalence of Staphylococci, S. aureus and MRSA in different food items that involve human handling.

\section{METHODS}

In present study total 1012 food samples were analyzed. These samples were provided by different food processing industries during 2013 to 2016.

\section{Isolation and Enumeration of Staphylococci and $S$. aureus}

Fifty grams of each sample were mixed with 450 $\mathrm{ml}$ of $0.1 \%$ peptone sterile physiological saline solution $(0.85 \% \mathrm{NaCl})$ and homogenized. Three decimal dilutions of each sample homogenate were prepared for enumeration of $S$. aureus. From each dilution, $0.3,0.3$ and $0.4 \mathrm{ml}$ was spread on Barid-Parker agar plates with egg yolk tellurite and were kept in an upright position until liquid was absorbed and then incubated at $35{ }^{\circ} \mathrm{C}$ for $24-48 \mathrm{~h}$. Typical black colonies with white zone were considered as $S$. aureus [5]. Staph Latex Kit (Prolix Latex Agglutination System) and growth on Mannitol Salt Agar (BioM), Staph-chromo agar (Merck), Staphylococcus 110 Agar (BioM), and Blood Agar (Oxoid) was used for further confirmation.

\section{Determination of plasma-coagulase and nuclease activity}

Colonies of staphylococci were individually reinoculated into test tubes containing $1.5 \mathrm{~mL}$ of Brain Heart Infusion Broth (BHI Broth, Oxoid). After $24 \mathrm{~h}$ of incubation at $35^{\circ} \mathrm{C}, 0.5 \mathrm{~mL}$ of each sample was added to another test tube containing $1 \mathrm{~mL}$ rabbit plasma (Merck). Inoculated test tubes were incubated at $35^{\circ} \mathrm{C}$. Formation of coagulum was considered as positive reaction. Results were evaluated after 1 , 2, 3, 6 and $24 \mathrm{~h}$. Each isolate was inoculated on the surface of DNase Agar (Oxoid) and was incubated at $35^{\circ} \mathrm{C}$. After $24 \mathrm{~h}$ of incubation, medium was flooded and acidified with $1 \mathrm{~N}$ hydrochloric acid, the DNA precipitated the turbidity of medium and clear zones around colonies indicated positive DNase reaction. The number of isolates that presented positive and negative results was recorded [10].

\section{Determination of MIC for oxacillin}

$\mathrm{BHI}$ Agar (Oxoid) was used to measure the oxacillin resistance level according to the guidelines of Clinical Laboratory Standard Institute (CLSI) [6]. Minimum inhibitory concentration (MIC) was re-confirmed by E-test using $A B$-Biodisk according to the manufacturer's instructions.

\section{Polymerase chain reaction (PCR)}

For molecular studies, genomic DNA was isolated by using the DNase Kit (Qiagen), following the manufacturer's instructions. PCR amplification of mecA genes was performed with an MWG Thermal Cycler in a volume of $50 \mu$ of Promega Master Mix. Primers, described previously [6], were used for amplification of $m e c A$, for SCCmec typing of MRSA isolates and for agr allele (I-IV) typing. 16S RNA was used as internal control for gene expression and specie identification.

\section{Ethical approval}

This study was approved by the institutional ethics committee.

\section{RESULTS}

Present study describes the presence of Staphylococci in different food products. Specially, foods that require considerable handling during preparation and that are kept at slightly elevated temperatures after preparation 
are selected and included in this study (Table 1). Total 1012 samples were analyzed, 723 (71.4\%) samples showed the presence of Staphylococci (Table 1, Figure 1).

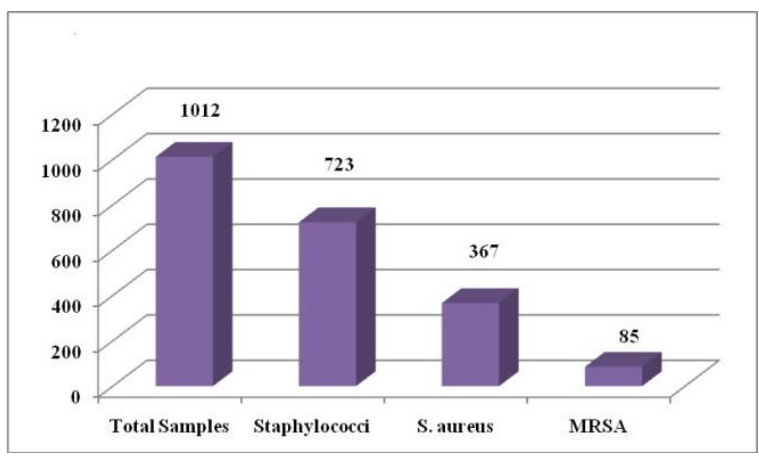

Figure 1. Isolation and identification of MRSA from food samples.

Out of 723 staphylococcal isolates, 367 (36.2\%) were S. aureus and $85(8.3 \%)$ isolates were confirmed as MRSA (Table 1, Figure 1). These isolates were identified on the basis of growth characters on selective and differential media. An isolate was considered within Staphylococcus genus when presented as gram-positive cocci in grape-like clusters, catalase, and mannitol fermentation positive. Coagulase test and growth on Baird-Parker agar and DNase agar confirmed the presence of $S$. aureus in subject food items. Positive reaction or amplification of $16 \mathrm{~s}$ and $\operatorname{mec} A$ gene specific primers confirm the presence of MRSA isolates in 85 food items. Out of 1012 samples 338 were spices mix; $80.77 \%$ of these samples showed the presence of staphylococci. The pathogenic $S$. aureus were present in $43.79 \%$ of spices mix samples with $5.03 \%$ of MRSA isolates. Among candy samples, $80.95 \%$ were positive for Staphylococci; $23.81 \%$ of these were $S$. aureus and $9.52 \%$ were MRSA. In fish and meat products, $40 \%$ and $87 \%$ showed Staphylococcal growth, respectively. In fish products $S$. aureus were $28 \%$ with $16 \%$ MRSA and in meat products $39 \%$ S. aureus with $7 \%$ MRSA. Samosa and Paratha (Frozen products) showed Staphylococci in $55 \%$ and $64 \%$ of samples, respectively. In Parathas $30.67 \%$ isolates were identified as $S$. aureus, in Samosa the $S$. aureus were $45 \%$, with $18.67 \%$ and $10 \%$, respectively MRSA. Rice and lentils respectively, showed $40.37 \%$ and $27.5 \%$ S. aureus with $9.17 \%$ and $7.5 \%$ MRSA. About $20 \%$ samples of Formula Milk and $10 \%$ of Mayonnaise samples were positive for MRSA. Table 1 depicts the complete details of samples and isolates. Moreover, molecular studies for MRSA typing showed that most of the isolates $(74.1 \%)$ belong to SCCmecA IV and 20\% MRSA isolates were SCCmecA type II and $5.8 \%$ isolates carry $S C C m e c A$ type III. Interestingly, isolates recovered from formula milk and mayonnaises were SCCmecA type II. The isolates that carry $S C C m e c A$ type III were recovered from meat products and candy samples. The MRSA isolates of SCCmecA type II belong to agr type I, while majority $(67 \%)$ of the isolates carry agr type II.

Table 1. MICs and typing of SCCmecA genes and agr in MRSA isolated from different food products.

\begin{tabular}{|c|c|c|c|c|c|c|c|c|c|c|c|c|}
\hline \multirow[t]{2}{*}{ Source } & \multirow{2}{*}{$\begin{array}{c}\text { No. of } \\
\text { Samples }\end{array}$} & \multirow{2}{*}{$\begin{array}{c}\text { Staphylococci, } \\
\text { n (\%) }\end{array}$} & \multirow{2}{*}{$\begin{array}{c}\text { S. aureus, } \mathbf{n} \\
(\%)\end{array}$} & \multirow{2}{*}{$\begin{array}{c}\text { MRSA, n } \\
(\%)\end{array}$} & \multicolumn{3}{|c|}{ MICs $(\mu / m \mathrm{l})$} & \multicolumn{2}{|c|}{$\begin{array}{c}\text { AGR } \\
\text { Typing }\end{array}$} & \multicolumn{2}{|c|}{$\begin{array}{c}\text { SCCmecA } \\
\text { Typing }\end{array}$} & \multirow[b]{2}{*}{ IV } \\
\hline & & & & & 32 & 64 & 128 & I & II & II & III & \\
\hline Candies & 105 & 85 (80.95) & $25(23.81)$ & $10(9.52)$ & 2 & 4 & 4 & 2 & 8 & 2 & 2 & 6 \\
\hline Candy mix & 50 & $40(80.00)$ & $19(38.00)$ & $4(8.00)$ & 0 & 4 & 0 & 0 & 4 & 4 & 0 & 0 \\
\hline Dates & 85 & $40(56.25)$ & $17(20.00)$ & $6(7.06)$ & 2 & 4 & 0 & 0 & 6 & 0 & 0 & 6 \\
\hline Fish Products & 25 & $10(40.00)$ & $07(28.00)$ & $4(16.00)$ & 0 & 0 & 4 & 0 & 4 & 0 & 0 & 4 \\
\hline Formula Milk & 15 & $10(66.67)$ & 08 (53.33) & $3(20.00)$ & 0 & 0 & 3 & 3 & 0 & 3 & 0 & 0 \\
\hline Lentils & 40 & 19 (47.50) & $11(27.50)$ & $3(7.50)$ & 2 & 1 & 0 & 1 & 2 & 0 & 0 & 3 \\
\hline Mayonnaise & 30 & $12(40.00)$ & 08 (26.67) & $3(10.00)$ & 0 & 3 & 0 & 3 & 0 & 3 & 0 & 0 \\
\hline Meat Products & 100 & $87(87.00)$ & $39(44.83)$ & $7(7.00)$ & 2 & 5 & 0 & 3 & 4 & 0 & 3 & 4 \\
\hline Paratha & 75 & $48(64.00)$ & $23(30.67)$ & $14(18.67)$ & 4 & 7 & 3 & 6 & 8 & 3 & 0 & 11 \\
\hline Rice & 109 & 77 (70.64) & 44 (40.37) & $10(9.17)$ & 0 & 5 & 5 & 2 & 8 & 0 & 0 & 10 \\
\hline Samosa & 40 & $22(55.00)$ & $18(45.00)$ & $4(10.00)$ & 4 & 0 & 0 & 4 & 0 & 2 & 0 & 2 \\
\hline Spices Mix & 338 & $273(80.77)$ & $148(43.79)$ & $17(5.03)$ & 17 & 0 & 0 & 0 & 17 & 0 & 0 & 17 \\
\hline
\end{tabular}

\section{DISCUSSION}

In the present study, commercial food items, those involve human handling, during processing and packaging, showed high rate of staphylococcal contamination, including $S$. aureus and MRSA. In present study it has been observed that food handlers are the major 
source of staphylococcal contamination. Although, the original source for staphylococcal introduction to food items was not traced out, even then this study creates doubt about food safety and highlights influence of food handling and processing on consumer health. Of more concern is the presence of MRSA; out of 1012 samples $367,(36.2 \%)$ were contaminated with S. aureus and $85(8.3 \%)$ with MRSA. This is a very serious situation. Due to multi-drug resistance and enterotoxins production, MRSA could be more fatal as compared to methicillin sensitive S. aureus. According to Jones at al [7] MRSA are as likely to produce enterotoxins as are methicillin-sensitive strains. Normally, it is considered that MRSA can survive in limited food items e.g. dairy products, meat and through cross contamination during kitchen processing of the recipe. However, during present study, recovery of MRSA from different variety of foods e.g. Lentils, Rice, Spices mix, Sweet mix and Dates, suggest the versatility of this pathogen. Moreover, majority of these isolates exhibited low-level oxacillin resistance (MIC ranges 32 to $128 \mu \mathrm{g} / \mathrm{ml}$ ), a character of the community of MRSA. According to Boyle-Vavra et al. [8], community-acquired MRSA (CAMRSA) isolates usually carry SCCmec type IV. Out of 87 subject isolates of MRSA, $63(72.4 \%)$ belong to SCCmec type IV. According to Song et al. [9] SCCmec type IV is the most predominant community type clone in the Asian countries. Interestingly, MRSA that belongs to SCCmec type II was recovered from sweet products only e.g. candies, mayonnaise, and formula Milk; these products involve more human handling as compared to the other products tested. The SCCmec type IV normally belongs to community acquired isolates of MRSA and SCCmec type II is associated with hospital acquired isolates. It is reported that SCCmec type II isolates are highly resistant type of MRSA, whereas SCCmec type IV exhibited low-level of resistance and are only resistant to ß-lactam antibiotics; as noticed in the present study. Although, this study is based on food items that involve human handling, but the true source of MRSA remains to be elucidated. Of more concern is to determine how these isolates of $S$. aureus develop oxacillin resistance. According to best of our knowledge, in Pakistan the use of antibiotics in agriculture is not common. However, use of antibiotics as a growth promoter in poultry industry is a common practice but good data are not available. So, this study which is based on wide range of samples provides us intimation about the prevalence rate of MRSA is our community.

\section{ACKNOWLEDGEMENT}

We are thankful to PCSIR Laboratories Complex Karachi and supporting staff of Microbiology for supporting this study.

\section{Funding:}

None.

\section{Competing interests:}

None to declare.

\section{REFERENCES}

1. Lambrechts AA, Human IS, Doughari JH, Lues JFR. Bacterial contamination of the hands of food handlers as indicator of hand washing efficacy in some convenient food industries in South Africa. Pak J Med Sci 2014; 30 (4):755-758.

2. Hennekinne JA, De Buyser ML, Dragacci S. Staphylococcus aureus and its food poisoning toxins: characterization and outbreak investigation. FEMS Microbiol Rev 2012; 36: 815836.

3. Kadariya J, Smith TC, Thapaliya D. Staphylococcus aureus and staphylococcal foodborne disease: An ongoing challenge in public health. BioMed Res Int 2014. doi: 10.1155/2014/827965.

4. David MZ, Daum RS. Community-associated methicillin-resistant Staphylococcus aureus: epidemiology and clinical consequences of an emerging epidemic. Clin Microbiol Rev 2010; 23 : 616-687.

5. Benntt W, Lancette GA. Bacteriological Analytical Manual (Chapter 12): Staphylococcus aureus. Department of Health and Human Services, $8^{\text {th }}$ edition. US Food and Drug Administration, 2001.

6. Mirani ZA, Aziz M, Khan MN, Lal I, Hassan NU, Khan SI. Biofilm formation and dispersal of Staphylococcus aureus under the influence of oxacillin. Microb Pathog 2013; 61-62C: 66-72.

7. Jones TF, Kellum ME, Porter SS, Bell M, Schaffner W. An outbreak of community-acquired foodborne illness caused by methicillin-resistant Staphylococcus aureus. Emerg Infect Dis 2002; 8: 82-84.

8. Boyle-Vavra S, Ereshefsky B, Wang CC, Daum RS. Successful multiresistant community- 
associated methicillin-resistant Staphylococcus aureus lineage from Taipei, Taiwan, that carries either the novel staphylococcal chromosome cassette mec (SCCmec) type VT or SCCmec type IV. J Clin Microbiol 2005; 43: 4719-4730.

9. Song JH, Hsueh PR, Chung DR, et al. (2011) Spread of methicillin-resistant Staphylococcus aureus between the community and the hospitals in Asian countries: an ANSORP study. J Antimicrob Chemother 2011; 66:1061-1069.

10. Koneman EWAS, Janda WM, Schreckenberger PC, Winn WC, In: The Gram positive cocci: Staphylococci and related organims. In Color Atlas and Textbook of Diagnostic Microbiology. 5. Koneman EW, Ed. Philadelphia: Lippincott-Raven; 1997; 551-576. 